\title{
The Voltage Controlled Oscillator with Coupled Microstrip Lines Resonator
}

\author{
Vyacheslav V. Babich*, \\ Bogdan A. Barabolya and Alexey V. Petukhov \\ Rostov-On-Don Research Institute of Radio Communication \\ 130 Nansena Str., Rostov-on-Don, 344038, Russia
}

Received 11.07.2018, received in revised form 13.07.2018, accepted 16.07.2018

The results of the development and research S-band voltage-controlled oscillator (VCO) with coupled microstrip lines resonator performed on the Russian components are presented. The device provides the low phase noise level, a wide tuning range and small physical size.

Keywords: voltage-controlled oscillator, Colpitts oscillator.

Citation: Babich V.V., Barabolya B.A., Petukhov A.V. The voltage controlled oscillator with coupled microstrip lines resonator, J. Sib. Fed. Univ. Eng. technol., 2019, 12(3), 275-283. DOI: 10.17516/1999-494X-0079.

\section{Генератор, управляемый напряжением, на связанных микрополосковых линиях}

\author{
В.В. Бабич, Б.А. Бараболя, А.В. Петухов \\ Ростовский-на-Дону \\ научно-исследовательский институт радиосвязи \\ Россия, 344038, Ростов-на-Дону, ул. Нансена, 130
}

Приведены результаты разработки и исследований генератора, управляемого напряжением (ГУН), S-диапазона на связанных микрополосковых линиях (МПЛ), выполненного на отечественной элементной базе. Устройство обеспечивает низкий уровень спектральной плотности мощности фазовых шумов (СПМФШ), широкий диапазон перестройки по частоте и имеет малые габаритные размеры.

Ключевые слова: генератор, управляемый напряжением, генератор Колпития.

Одним из основных элементов синтезаторов частот, применяемых в передающих и приемных трактах радиосистем, является генератор сигналов, частота которого управляется внешним напряжением (ГУН).

(C) Siberian Federal University. All rights reserved

* Corresponding author E-mail address: A-z97@yandex.ru 
Двумя наиболее критичными параметрами ГУН для синтезаторов считаются диапазон перестройки и спектральная плотность мощности фазовых шумов [1,2].

В то время как номенклатура импортных микросхем ГУН достаточно обширна, ГУН отечественного производства практически не представлены. Применение отечественной комплектации устраняет ограничения, неизбежно появляющиеся при использовании продукции зарубежного производства. Таким образом, задача разработки ГУН на отечественной элементной базе актуальна.

Как правило, схема ГУН состоит в основном из частотно-избирательной цепи и активного усилительного устройства.

Фазовые шумы ГУН определяются применяемым транзистором и добротностью частотноизбирательной цепи. Высокодобротные резонаторы, такие как керамический, на поверхностных акустических волнах, диэлектрический, на железоиттриевом гранате, оптоэлектронный, громоздкие трехмерные структуры не подходят для многих современных применений. Помимо габаритов, такие резонаторы значительно ограничивают полосу перестройки и крайне чувствительны к вибрационным нагрузкам.

Цель работы - разработка ГУН на отечественной элементной базе, устойчивого к температурным и вибрационным воздействиям и не уступающего по своим характеристикам аналогичным ГУН зарубежного производства.

Решаемые задачи:

1. Разработка схемы ГУН на микрополосковой линии на отечественной элементной базе, обеспечивающей широкий диапазон перестройки и низкие фазовые шумы.

2. Анализ результатов экспериментального исследования характеристик макета ГУН.

Проведено исследование существующих типов МПР (микрополосковых резонаторов), а также моделирование и расчет их добротности с учетом материала подложки.

По абсолютному определению, собственная (ненагруженная) добротность резонатора $(Q)$ вычисляется как отношение энергии, накопленной в резонаторе, к энергии, рассеиваемой за период на резонансной частоте. Однако для упрощения анализа $Q$ можно оценить графически с использованием расчета ширины полосы пропускания по ЗдБ (1):

$$
Q=\frac{f_{0}}{B W}=\frac{f_{0}}{f_{H}-f_{L}},
$$

где $f_{0}$ - центральная частота; $B W$ - полоса пропускания по 3 дБ; $f_{H}-$ верхняя частота; $f_{L}-$ нижняя частота.

На рис. 1 представлен график определения добротности для МПР на связанных линияX.

Учитывая, что на добротность линии влияют потери энергии в диэлектрике, с использованием формулы расчета добротности по мощности были измерены значения добротности при различных значениях тангенса угла диэлектрических потерь (в диапазоне [0,005; 0,03], табл. 1).

Таблица 1 показывает, что наилучшими значениями добротностей обладает МПР на связанных линиях при значениях тангенса диэлектрических потерь в диапазоне от 0.005 до 0,01 (рис. 2).

$$
-276-
$$




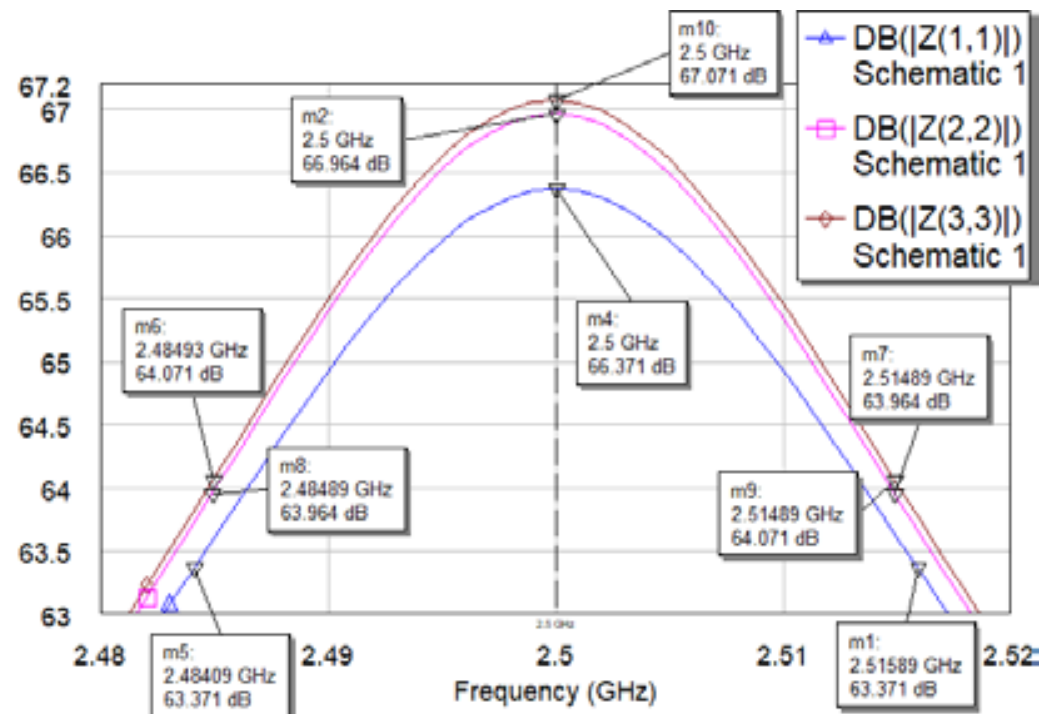

Рис. 1. График расчета добротности для микрополоскового резонатора

Fig. 1. Plot of $Q$-factor calculation of microstrip resonator

Таблица 1. Добротности МПР в зависимости от тангенса диэлектрических потерь

Table 1. $Q$-factor of microstrip resonator depending on loss tangent

\begin{tabular}{|c|c|c|c|}
\hline \multirow{2}{*}{$\begin{array}{c}\text { Значение тангенса } \\
\text { диэлектрических } \\
\text { потерь, tg } \delta\end{array}$} & $\begin{array}{c}|c| \\
\text { Мнкрополосковый } \\
\text { резонатор }\end{array}$ & $\begin{array}{c}\text { МПР на связанных } \\
\text { линиях (2-х) }\end{array}$ & $\begin{array}{c}\text { МПР на связанных } \\
\text { линиях (3-х) }\end{array}$ \\
\hline 0,005 & 124,37 & 130,89 & 132,27 \\
\hline 0,01 & 78,12 & 83,3 & 86,2 \\
\hline 0.015 & 56,81 & 60,67 & 60,97 \\
\hline 0,02 & 45,45 & 47,8 & 48,07 \\
\hline 0,025 & 37,31 & 39,43 & 39,74 \\
\hline 0,03 & 32,05 & 33,6 & 33,73 \\
\hline
\end{tabular}

Таким образом, одним из возможных решений выступает использование резонатора на связанных МПЛ [3].

Непосредственным плюсом резонатора на МПЛ является то, что его физический размер может быть уменьшен путем изменения фазовой скорости волны в линии. Уменьшение фазовой скорости приводит к соответствующему уменьшению длины волны (2). В иностранной литературе этот прием носит название «эффект медленной волны» [3]. Данный эффект может быть достигнут путем добавления в МПЛ периодических емкостных нагрузок.

$$
v_{\phi}=f \times \lambda=\frac{c_{0}}{\sqrt{\mu_{r} \varepsilon_{r}}},
$$


где $v_{\phi}$ - фазовая скорость волны; $\lambda$ - длина волны в линии передачи; $f$ - частота колебаний; $c_{0}$ - скорость света в вакууме; $\mu_{r}$ - относительная магнитная проницаемость; $\varepsilon_{r}-$ относительная диэлектрическая проницаемость.



Рис. 2. Зависимость добротности линии от диэлектрических потерь линии

Fig. 2. $Q$-factor of microstrip line depending on loss tangent

Линию передачи можно описать характеристическим импедансом $Z_{0}$ линии, фазовой скоростью распространения волны $v_{\phi}$ и фазовой постоянной $\beta$, численно равной сдвигу фазы волны в отрезке линии передачи единичной длины:

$$
\begin{aligned}
& Z_{0}=\sqrt{\frac{L}{C}}, \\
& v_{\phi}=\frac{1}{\sqrt{\mu \varepsilon}}=\frac{1}{\sqrt{L C}} \Rightarrow L C=\mu \varepsilon, \\
& v_{\phi}=\frac{\beta}{\omega} \Rightarrow \beta=\omega \sqrt{L C},
\end{aligned}
$$

где $\mu, \varepsilon$ - абсолютная магнитная и диэлектрическая проницаемости; соответственно, $\omega$ - циклическая частота.

Из (4) видно, что для заданных $\mu$ и $\varepsilon$ невозможно уменьшить $v_{\phi}$ в регулярной линии передачи путем увеличения либо индуктивности, либо емкости на единицу длины, поскольку увеличение индуктивности $L$ приведет к уменьшению емкости $C$ и наоборот.

Устранив ограничение, что линия должна быть регулярной, эффективное увеличение емкости на единицу длины становится возможным без уменьшения индуктивности. Это может быть реализовано путем нагрузки печатной линии шунтирующей емкостью с периодическими интервалами (рис. 3). Данная структура будет проявлять эффект «медленной волны» за счет уменьшения фазовой скорости $v_{\phi}(4)$. В свою очередь, за счет увеличения постоянной распространения $\beta$ (5) резонатор с использованием эффекта медленной волны не только приобретает компактный размер, но и проявляет высокую частотную селективность. 
Это позволяет использовать его в ГУН с требованием к уровню СПМФШ в спектре выходного сигнала.

Схема ГУН построена на основе емкостной трехточки [4] с использованием в качестве активного элемента отечественного биполярного транзистора (рис. 4).

Частотно-избирательная цепь разработана на основе микрополоскового резонатора на связанных линиях с эффектом медленной волны. Перестройка по частоте осуществляется эпитаксиально-планарными варикапами. Данная схема была настроена и оптимизирована на рабочую частоту 2.55 ГГц. Добротность обычной МПЛ со связанными линиями при длине 13,55 мм и ширине 3,3 мм равна 39, а МПЛ со связанными линиями с периодическими емкостями нагрузки равна 110 при длине 10,2 мм и ширине 3 мм (значение тангенса угла диэлектрических потерь равно 0,025). Достигнуто улучшение добротности МПЛ резонатора со связанными линиями с периодическими емкостями нагрузки почти в 3 раза, следовательно, такой резонатор можно считать пригодным для использования в разрабатываемой схеме ГУН.


Рис. 3. Схема периодически нагруженной МПЛ передачи. C_Load - периодические емкости нагрузки

Fig 3. Topology of microstrip line periodically loaded by shunt capacitors C_Load

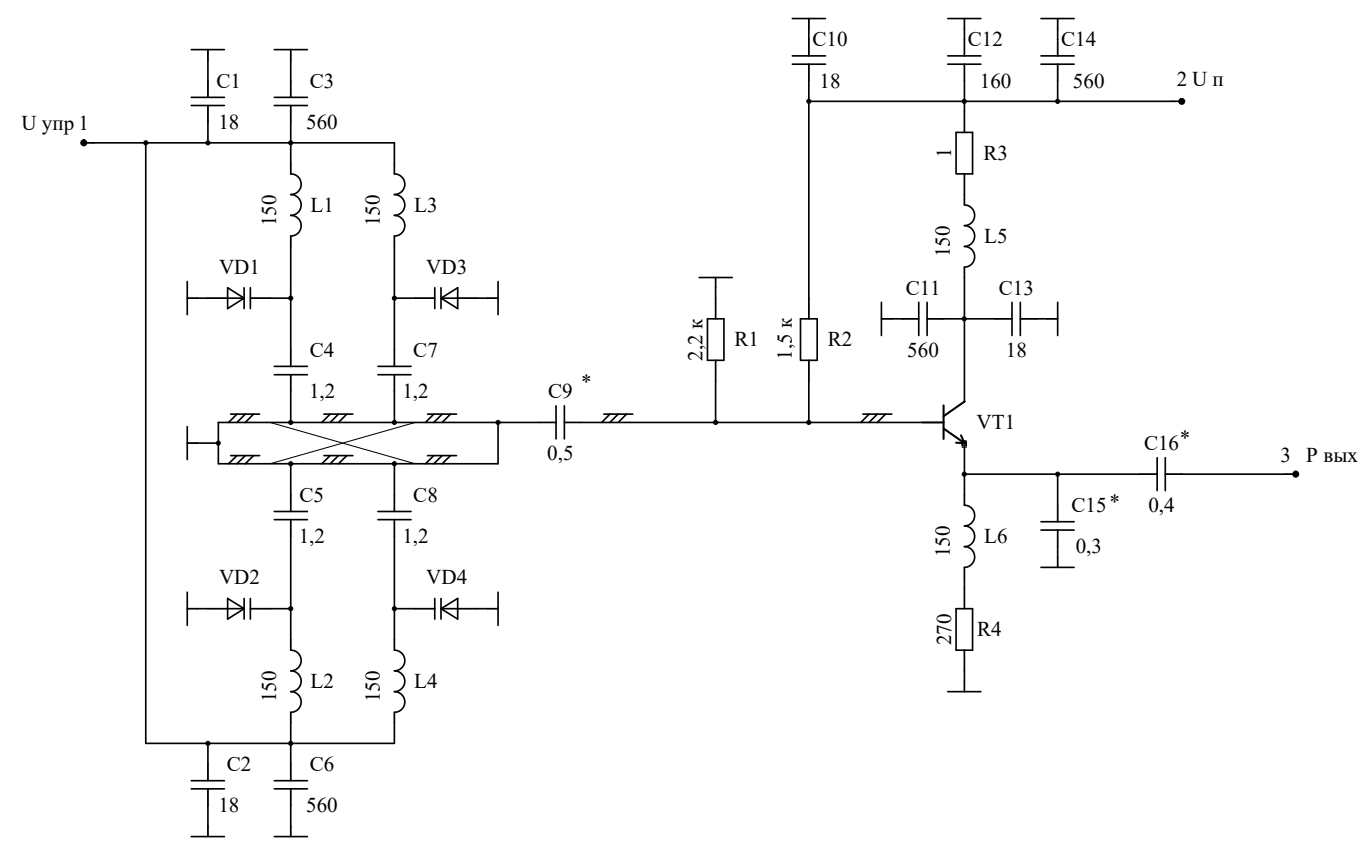

Рис. 4. Электрическая схема ГУН

Fig. 4. Schematic of a voltage-controlled oscillator 
Разработка схемы ГУН включает два последовательных этапа. Первый - это линейный анализ, суть которого заключается в том, чтобы найти приблизительную частоту генерации, исходя из условия соблюдения баланса фаз и амплитуд. Затем найденная частота используется в качестве нулевой точки второго этапа - нелинейного анализа, в процессе которого рассчитываются основные характеристики ГУН: спектр, спектральная плотность мощности фазовых шумов (СПМФШ), зависимость частоты от напряжения управления и форма выходного сигнала.

По рассчитанной схеме собран и исследован макет ГУН на связанных МПЛ. Измерены основные параметры и проведен сравнительный анализ с ГУН зарубежного производства (рис. 5, 6).

Всплески фазовых шумов (рис. $6 a$ ) обусловлены собственными помехами измерительного устройства и исчезают при использовании хорошей экранировки самого ГУН, а также при достаточной фильтрации питающего напряжения, что подтверждено экспериментально.

Разработанный ГУН обеспечивает устойчивость к воздействию синусоидальной и широкополосной случайной вибрации, а также к воздействию температур в широком диапазоне: от минус 60 до $60{ }^{\circ} \mathrm{C}$. При испытании на воздействие повышенной температуры происходит ухудшение СПМФШ на 3 дБ.

Специфика применения комплектации отечественного производства заключается в том, что, во-первых, номенклатура очень ограничена; во-вторых, основные параметры используемых компонентов могут варьироваться в больших пределах, что усложняет как расчеты, так и последующую настройку ГУН; в-третьих, отечественные производители транзисторов не предоставляют эквивалентные схемы для проведения расчетов.

Полученные характеристики разработанного ГУН сравнимы с аналогичными характеристиками ГУН зарубежного производства. Их сравнение представлено в табл. 2.

Исследования макета показали высокую вибрационную стойкость исследуемого ГУН. Минимальная рабочая температура приведенных в табл. 2 импортных аналогов ограничена производителем значением минус $40^{\circ} \mathrm{C}$. Также не регламентирована требуемая вибрационная устойчивость. Таким образом, разработанный ГУН не уступает по характеристикам импортным аналогам и при этом обеспечивает устойчивую работу в температурном диапазоне от минус 60 до $60{ }^{\circ} \mathrm{C}$ и при повышенных вибрационных воздействиях.

Таблица 2. Сравнение разработанного ГУН и косвенных зарубежных аналогов Table 2. Comparison of developed VCO and foreign analogues

\begin{tabular}{|c|c|c|c|c|c|c|c|c|}
\hline & \multicolumn{2}{|c|}{ Частота, МГц } & \multirow{2}{*}{$\begin{array}{c}\mathrm{U}_{\text {упр }}, \\
\mathrm{B}\end{array}$} & \multirow{2}{*}{$\begin{array}{c}\mathrm{U}_{\text {пит }}, \\
\mathrm{B}\end{array}$} & \multirow{2}{*}{$\begin{array}{c}\mathrm{I}_{\text {пит, }} \\
\text { мА }\end{array}$} & \multirow{2}{*}{\begin{tabular}{|c|} 
Крутизна, \\
МГц/B
\end{tabular}} & \multicolumn{2}{|c|}{ СПМФШ, дБн/Гц } \\
\hline & Нижняя & Верхняя & & & & & 10 кГц & $100 \kappa \Gamma_{ц}$ \\
\hline $\begin{array}{l}\text { Разработанный } \\
\text { ГУН } \\
\text { «ФГУП РНИИРС» }\end{array}$ & 2420 & 2720 & $\begin{array}{l}\text { от } 0 \\
\text { до } 20\end{array}$ & 8 & 15 & $\begin{array}{l}\text { от } 10 \\
\text { до } 28\end{array}$ & -102 & -123 \\
\hline \begin{tabular}{|l|} 
DCRO240270-5 \\
Synergy Microwave
\end{tabular} & 2400 & 2700 & $\begin{array}{l}\text { от } 0,5 \\
\text { до } 12\end{array}$ & 5 & 23 & $\begin{array}{l}\text { от } 28 \\
\text { до } 38\end{array}$ & -103 & -124 \\
\hline \begin{tabular}{|l|} 
DCO225275-5 \\
Synergy Microwave
\end{tabular} & 2200 & 2750 & $\begin{array}{l}\text { от } 0,5 \\
\text { до } 12\end{array}$ & 5 & 22 & $\begin{array}{l}\text { от } 35 \\
\text { до } 85\end{array}$ & -95 & -117 \\
\hline
\end{tabular}




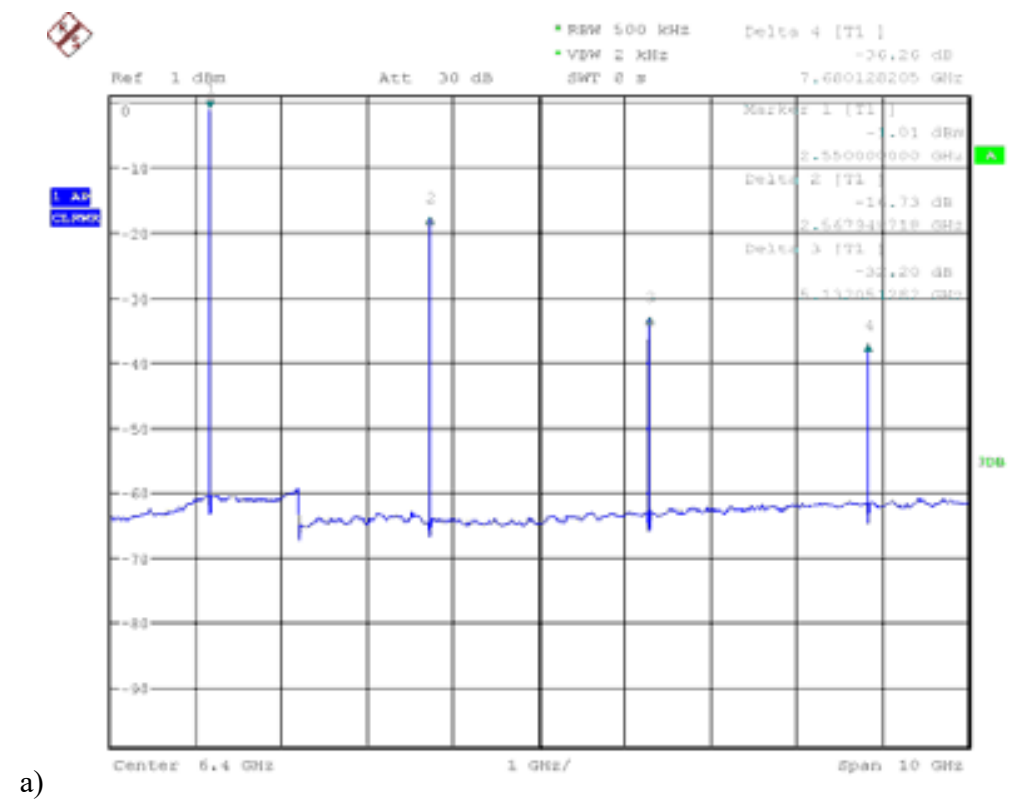

a)

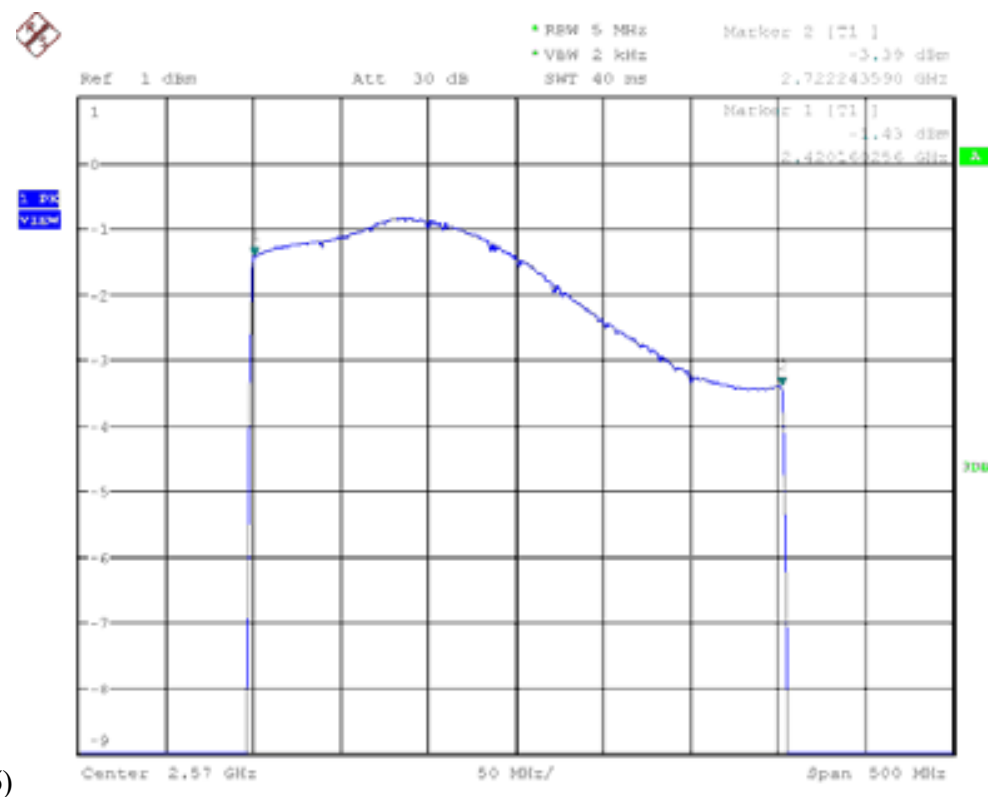

Рис. 5. Характеристики выходного сигнала ГУН: а - спектр выходного сигнала ГУН; б - неравномерность выходного сигнала ГУН

Fig. 5. Shows the measured VCOs characteristics: $a-$ spectra; $\sigma$ - output power 


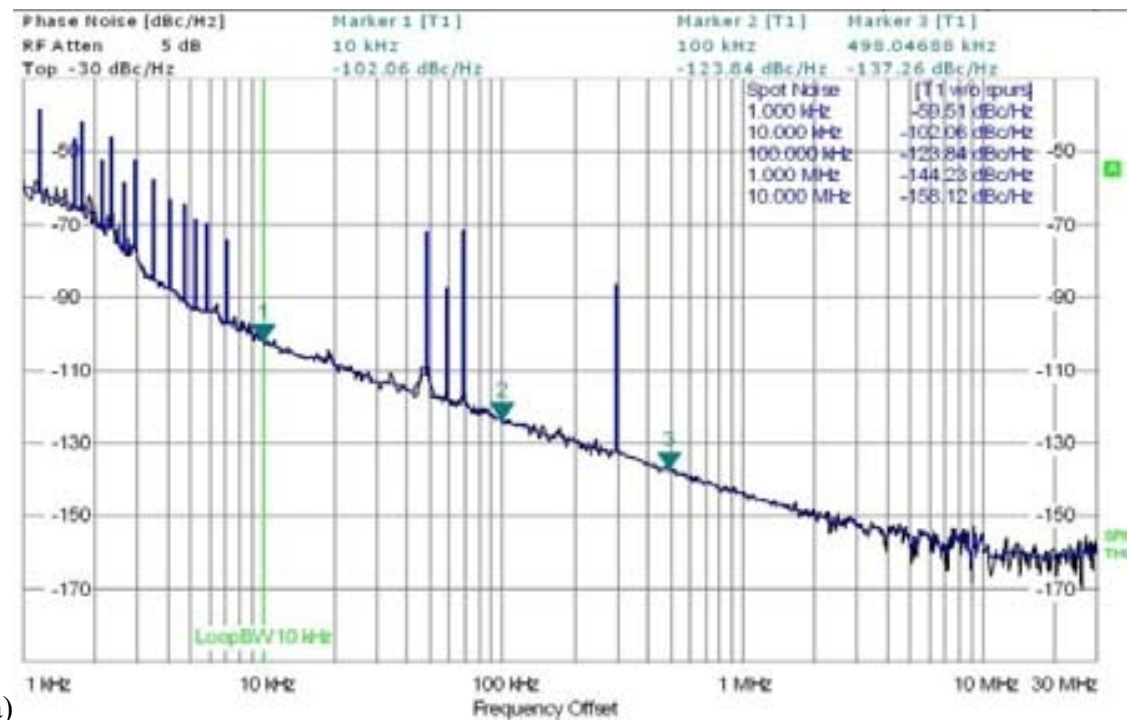

a)

Fequency Otreet

$10 \mathrm{MHe}$. $30 \mathrm{MHe}$

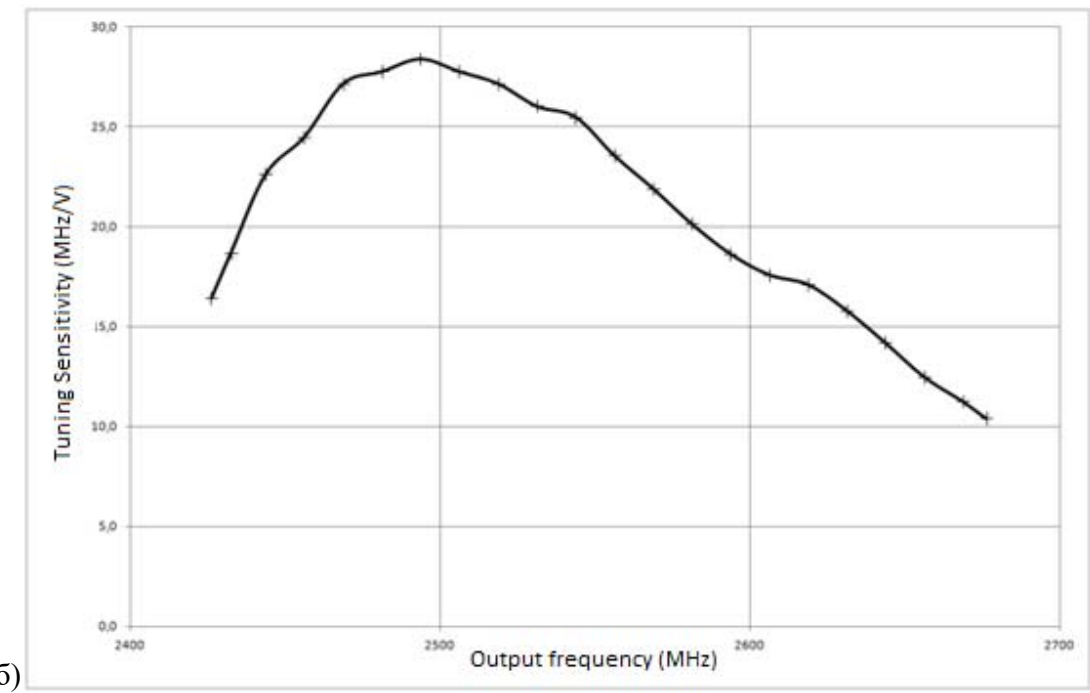

Рис. 6. Характеристики выходного сигнала ГУН: а - СПМФШ ГУН на центральной частоте 2,55 ГГц; б зависимость крутизны перестройки ГУН от частоты выходного сигнала

Fig. 6. Shows the measured VCOs characteristics: a - the oscillator phase noise at oscillation frequency 2,55 GHz; $\sigma$ - tuning sensitivity

\section{Выводы}

1. Разработанная схема ГУН с использованием эффекта «медленной волны» путем шунтирования микрополоскового резонатора обеспечивает диапазон перестройки не менее 9,5% с уровнем СПМФШ - 123 дБн/Гц при отстройке от несущей частоты в 100 кГц.

2. Экспериментальные исследования показали, что разработанный ГУН обеспечивает устойчивую работу:

- в температурном диапазоне от минус 60 до $60{ }^{\circ} \mathrm{C}$, что превосходит известные импортные аналоги; 
- при воздействии широкополосной случайной вибрации с перегрузкой в среднем $10 \mathrm{~g}$.

\section{Список литературы}

[1] Манассевич В. Синтезаторы частот. Теория и проектирование. Москва: Связь, 1979, 384 c. [Manassevich V. Frequency synthesizers. Theoryanddesign. Moscow: Communication, 1979, 384 p. (in Russian)].

[2] Рыжков А.В., Попов В.Н. Синтезаторы частот в технике радиосвязи. Москва: Радио и связь, 1991, 264 с. [Ryzhkov A.V., Popov V.N. Frequency synthesizers in radio communication technique. Moscow: radio and communication, 1991, 264 p. (in Russian)].

[3] Dr.-Ing. Ajay K. Poddar. Slow Wave Resonator Based Tunable Multi-Band Multi-Mode Injection-Locked Oscillators. Brandenburg University of Technology Cottbus-Senftenberg, 2013, $586 \mathrm{p}$.

[4] Cornelis J. Kikkert. RF Electronics: Design and Simulation. James Cook University Townsville, Queensland, Australia, 2015 Edition, 309 p. 